\title{
Eine Bodenwertsteuer als Grundsteuer?
}

\begin{abstract}
Nach dem Kompromiss von Bundestag und Bundesrat über die Reform der Grundsteuer Ende 2019 erhalten die Länder Entscheidungsspielraum darüber, ob sie sich dem Bundesmodell anschließen oder ein eigenes Steuermodell entwickeln. Ein baden-württembergischer Gesetzentwurf sieht eine Bodenwertsteuer als alleinige Grundsteuer vor. Er verzichtet auf einen Gebäudeteil in der Grundsteuer, der in anderen Modellen und im Bundesvorschlag enthalten ist. Eine reine Bodenwertsteuer weist gegenüber dem Bundesvorschlag und anderen Vorschlägen deutliche Vorteile bei den Erhebungskosten auf. Allerdings führt der Verzicht auf den Gebäudeteil dazu, dass die Nutzung der Grundstücke nicht erfasst wird. Zudem ergeben sich aus der einseitigen Belastung der Grundstückseigentümer politische Risiken, die durch Vorgabe eines Höchsthebesatzes begrenzt werden sollten.
\end{abstract}

\begin{abstract}
Eine Kommunalsteuer, die ausschließlich nach dem Bodenwert bemessen wird und Gebäude nicht berücksichtigt, spielte bisher in der politischen Reformdiskussion zur Grundsteuer keine wichtige Rolle. Sie wurde zwar bei der Erörterung der verschiedenen Vorschläge mitgeführt, ${ }^{1}$ aber eigentlich rechnete kaum jemand damit, dass diese Variante einmal ernsthaft Gegenstand eines Reformansatzes werden könnte. Das änderte sich spätestens, als das Finanzministerium des Landes Baden-Württemberg im November 2019 einen Entwurf zur Grundsteuerreform vorlegte, der genau dies tat: Bemessungsgrundlage soll
\end{abstract}

(C) Der/die Autor(en) 2020. Open Access: Dieser Artikel wird unter der Creative Commons Namensnennung 4.0 International Lizenz (https:// creativecommons.org/licenses/by/4.0/deed.de) veröffentlicht.

Open Access wird durch die ZBW - Leibniz-Informationszentrum Wirtschaft gefördert.

1 So erscheint sie in der zeitnahen Analyse von Scheffler und Roith (2018) als eine von acht Varianten für die Reform.

Prof. Dr. Thiess Büttner ist Professor für öffentliche Finanzen an der Friedrich-Alexander-Universität Erlangen-Nürnberg und Mitglied des Wissenschaftlichen Beirats beim Bundesministerium der Finanzen.

Prof. Dr. Dr. h.c. Horst Zimmermann ist emeritierter Professor für öffentliche Finanzen an der PhilippsUniversität Marburg und Mitglied des Wissenschaftlichen Beirats beim Bundesministerium der Finanzen. allein der Boden sein, wiedergegeben durch den Bodenrichtwert. Daher ist es nunmehr nötig, diesen neuen Tatbestand auch finanzwissenschaftlich zu reflektieren, denn derzeit sind viele Länder dabei, eine Entscheidung zu ihrer Grundsteuer zu treffen.

Bodenwertsteuer aus finanzwissenschaftlicher Sicht

Aus dem Blickwinkel der Finanzwissenschaft hat die Besteuerung von Grundstücken eine zentrale Stellung im System der Kommunalsteuern. Dies rührt vor allem daher, dass sich Steuern auf Grundstücke - anders als etwa Ertragsteuern - nicht auf eine mobile Bemessungsgrundlage mit entsprechenden Ausweichmöglichkeiten beziehen. Vielmehr belasten solche Steuern den Grundstückseigentümer. Durch Verlagerung des Wohnsitzes oder Betriebes kann der Eigentümer der Last nicht entgehen. Daher kommt es nicht zu Ausweichreaktionen und zu keiner Verzerrung der Standortwahl. Insofern sind Steuern auf das Grundstück eine robuste Finanzierungsquelle für Gemeinden, die sich einem intensiven Standortwettbewerb ausgesetzt sehen. Dies gilt auch für die Bodenwertsteuer, die sich ausschließlich auf den Wert des Grundstücks bezieht. Sie ist daher ein attraktives Finanzierungsinstrument für Gemeinden.

Im Hinblick auf die Steuergerechtigkeit und damit unter Verteilungsgesichtspunkten ist die Bodenwertsteuer allerdings nur bedingt zu rechtfertigen. Sie folgt vor allem nicht dem Prinzip der Besteuerung nach der Leistungsfähigkeit. Denn erstens knüpft sie am Objekt und nicht an der Person an, und zweitens ist sie eine Bruttovermögensteuer und berücksichtigt nicht etwaige für den Erwerb des Objektes aufgenommene Schulden. Letztlich kann eine Rechtfertigung der Bodenwertsteuer nur aus dem Äquivalenzprinzip erfolgen (Zimmermann und Döring, 
2019). ${ }^{2}$ Bei dieser Sichtweise ist die Bodenwertsteuer das finanzielle Gegenstück, ein „Äquivalent“, für den Nutzen aus Gemeindeleistungen, die dem Grundstück und seinem Eigentümer direkt oder indirekt zugutekommen.

Dieser Nutzen ist ein grober, aber dennoch gewichtiger Maßstab. Um sich die Bedeutung vor Augen zu führen, denke man an zwei gleiche mittelgroße Städte in ähnlicher regionaler und struktureller Situation. Beide Städte weisen den gleichen Hebesatz bei der Bodenwertsteuer aus. Die eine Stadt gilt als gut verwaltet. Ihre Städtebaupolitik hat für eine gute Mischung aus bebauten und offenen Zonen gesorgt. Auch ist die Erreichbarkeit der Stadt und ihrer Umgebung durch die Verkehrspolitik gesichert. Das Schulwesen ist ebenso wie die anderen Infrastrukturbereiche der Stadt in einem sehr guten Zustand. In der anderen Stadt ist es genau umgekehrt, und das ist bekannt. Wer zwischen beiden Städten entscheiden kann, weil er in beiden ein Stellenangebot hat, wird die zuerst genannte Stadt bevorzugen. Dort steigen dementsprechend die Grundstückspreise, denn sie spiegeln diese Wirkungen der Infrastruktur wider. Wenn der höhere Grundstückswert sich in einer am Wert orientierten Bemessungsgrundlage niederschlägt, steht den besseren Leistungen trotz des gleichen Hebesatzes auch eine höhere Steuerlast in dieser Stadt gegenüber. Eine am Wert des Grundstücks anknüpfende Steuer kann sich sehr dynamisch entwickeln. So können Änderungen in der Wertigkeit der Grundstücke innerhalb einer Gemeinde oder zwischen Gemeinden zu Anpassungen in der Steuerlast führen. Die Berücksichtigung solcher Anpassungen bei der Besteuerung ist im Sinne des Äquivalenzprinzips, soweit sie Unterschiede in der Standortqualität reflektieren.

Allerdings schlagen sich auch andere Entwicklungen bei den Grundstückspreisen in der Grundsteuer nieder. Hieraus resultierende Steuerlastveränderungen sind mit dem Äquivalenzprinzip nicht zu rechtfertigen. Fällt etwa die allgemeine Preisentwicklung bei den Grundstücken stärker aus als bei anderen Gütern, beispielsweise aufgrund der Zinsentwicklung, kann es zu einem starken Anstieg der Belastung kommen, ohne dass das Angebot an öffentlichen Leistungen notwendigerweise wächst. Diese Problematik hat sich bei der US-amerikanischen „Property Tax" gezeigt. Als es in den 1970er Jahren zu massiven Preissteigerungen bei Immobilien kam, wurden in vielen Bundesstaaten die Rechte der Gemeinden und vor allem der für die Schulen verantwortlichen „School Districts“ auf die Festsetzung der Steuerbelastung eingeschränkt. Heute haben 46 von 50 Bundesstaaten Vorschriften zur Begrenzung der Steuerbelastung mit der „Property Tax“ (Walczak, 2018).

2 Die Begründung der Grundsteuer als Äquivalenzsteuer ist auch im Verfassungsrecht bekannt, wenn auch nur als eine von mehreren (in allgemeiner Form Hey, 2019, 308 und 321 speziell zur Bodenwertsteuer).
Schwierigkeiten bei einer Rechtfertigung anhand des Äquivalenzprinzips entstehen auch beim verfassungsrechtlich garantierten Hebesatzrecht. Anders als bei Gebühren und Beiträgen ist die Gemeinde nicht dazu gezwungen, die mit einem Anstieg des Hebesatzes erzielten Mehreinnahmen für Projekte zu verwenden, die den Grundstückseigentümern direkt oder indirekt einen Nutzen stiften. Daher ist die Bodenwertsteuer auch ein Instrument zur einseitigen Belastung von Grundstückseigentümern, da sie langfristig nicht überwälzt werden kann. Radikale Steuerreformer in der Tradition von Henry George befürworten entsprechend eine vollständige Enteignung des scheinbar „leistungslosen Vermögens“ auf dem Wege der Besteuerung (van Suntum und Schultewolter, 2014).

\section{Bedeutung des Gebäudeelements}

In ihrer heutigen Form berücksichtigt die Grundsteuer nicht nur den Grundstückswert, sondern auch die Nutzung des Grundstücks durch Bezug auf den Gebäudewert. Dieser zweiten Komponente der Bemessungsgrundlage wird in der finanzwissenschaftlichen Literatur zu den Kommunalfinanzen eine wichtige Funktion beigemessen. Durch die Einbeziehung des Gebäudewerts reflektiert die Steuerbelastung auch die zusätzlichen Kosten, die der Gemeinde durch intensivere Nutzung der kommunalen Leistungen entstehen (Wissenschaftlicher Beirat beim Bundesministerium der Finanzen, 2011). Zur Illustration stelle man sich zwei gleich große Grundstücke in ähnlicher Lage vor. Das eine sei mit einem Einfamilienhaus bebaut und das andere mit einem Miethaus mit mehreren Parteien. Durch die Berücksichtigung des Gebäudeteils wird die stärkere Inanspruchnahme gemeindlicher Leistungen durch die zahlreicheren Bewohner des zweiten Grundstücks auch in einer höheren Steuerbelastung abgebildet. ${ }^{3}$

Vor diesem Hintergrund fordert der Wissenschaftliche Beirat in seiner Stellungnahme zur Reform der Grundsteuer, die Kombination von Grundstückswert und Gebäudeteil bei der Reform der Grundsteuer beizubehalten. ${ }^{4}$ Die Berücksichtigung des Gebäudeteils ist nicht nur wegen der Nutzung des Grundstücks von Bedeutung. Durch die Einbeziehung des Gebäudeteils würde auch eine einseitige Belastung der Grundstückseigentümer erschwert. Ein starker Anstieg im Hebesatz würde in diesem Fall teils auf die Nutzer des Grundstücks überwälzt (Wissenschaftlicher Beirat beim Bundesministerium der Finanzen, 2011, 93).

3 Hier steht das Äquivalenzprinzip im Konflikt mit der Lenkungswirkung einer Bodenwertsteuer als Anreiz für die Verdichtung (Löhr, 2019).

4 Der Wissenschaftliche Beirat legt Wert darauf, dass die beiden Komponenten nicht unabhängig voneinander festgelegt werden. Der Kronberger Kreis (2003) demgegenüber bevorzugt eine frei wählbare Kombination von Bodenwertsteuer und allgemeiner Bürgersteuer. 


\section{Vorteil der leichten Administrierbarkeit}

Die Literatur zur Grundsteuer betont, dass vor allem die Bewertung des Gebäudeteils Probleme für die Steuerverwaltung aufwirft. Scheffler und Roith (2018) sehen daher in der Administrierbarkeit bei der Bodenwertsteuer einen wichtigen Vorteil. Hier bietet sich im Vergleich zu der Anknüpfung am Mietwert eine vergleichsweise einfache Bewertung an, da lediglich die Informationen aus dem Kataster und die Bodenrichtwerte zusammengeführt werden müssen. Diese Vorgänge lassen sich zweifellos kostengünstig automatisieren, denn aus Sicht der Verwaltung ginge es nur darum, für den einzelnen Steuerbescheid aus dem Grundbuch außer dem Eigentümer die Quadratmeterzahl des Grundstücks zu entnehmen, diese mit dem vorhandenen Bodenrichtwert zu multiplizieren und darauf den im Formular vorgegebenen $\mathrm{He}$ besatz der Gemeinde anzuwenden, um den Steuerbescheid fertigzustellen. Dieses einfache Verwaltungsverfahren hat zudem den Vorteil, dass die Veranlagungszeiträume kurz gehalten werden können. Die Quadratmeter sind zumeist die gleichen geblieben, sodass nur die geänderten Bodenrichtwerte neu eingesetzt werden müssten. Ebenfalls zu niedrigen Verwaltungskosten trägt bei, dass keine Angaben des Steuerzahlers nötig sind. Das erspart die Bereitstellung von Informationen durch den Steuerzahler und die Überprüfung dieser Daten. Dies ist ein großer Unterschied zu jeglichem Erklärungsverfahren, bei dem der Steuerzahler sich äußern muss. Die Erhebungskosten des Finanzamts sind also im Vergleich zu den Alternativen besonders niedrig. ${ }^{5}$

Es gibt Vorbehalte gegen die Genauigkeit der Bodenrichtwerte, etwa weil sie nicht für jedes Grundstück vorhanden sind, sondern nur für regionale Gruppen von Grundstücken. Ihre Qualität ist aber nicht zuletzt dadurch gesichert, dass sie auch für die Erbschaftsteuer verwendet werden. Bei der Erbschaftsteuer handelt es sich zwar im Vergleich zur Grundsteuer um jeweils wenige Fälle, aber dafür ist der behandelte Steuerbetrag oft sehr hoch. Es handelt sich aber, wie sich bei Rücksprache in hessischen Bewertungsstellen herausstellte, in aller Regel um nicht streitanfällige Angaben. Sicherlich müssen die Bodenrichtwerte im Gebiet des jeweiligen Landes an der einen oder anderen Stelle nachgebessert werden, aber bis zur konkreten Erhebung der reformierten Grundsteuer ist dafür noch Zeit.

\section{Praktikable Alternativen zur Bodenwertsteuer?}

In der Reformdiskussion wurde auch der Vorschlag gemacht, ganz auf Wertansätze zu verzichten. Im Zentrum

5 Der Aufwand für die Finanzverwaltung dürfte daher unter dem Ansatz bleiben, der für die Grundsteuerreform ursprünglich veranschlagt wurde. Baden-Württemberg hatte für die IT-Kosten der Grundsteuerreform - noch ohne Bezug zur Bodenwertsteuer - einen „mittleren zweistelligen Millionenbetrag“ veranschlagt (Bundesrat, 2019b). steht das sogenannte Südländermodell der Flächensteuer. Hier werden die Flächen des Grundstücks und die Gebäudefläche zugrunde gelegt, aber ohne Wertbezug (Fuest et al., 2018). Die Verwaltungskosten wären vermutlich höher als bei der Bodenwertsteuer. Es müssen zwar keine Bodenrichtwerte, dafür aber die Gebäudeflächen erhoben werden. Unter Wirkungsaspekten hätte diese Steuer den Nachteil, dass Unterschiede in der Standortqualität nicht berücksichtigt werden: Teure attraktive und ungünstig gelegene preiswerte Grundstücke würden gleich besteuert.

Zudem würden Preisentwicklungen, die eine geänderte Standortqualität reflektieren, nicht in die Besteuerung einfließen. Befürworter der Flächensteuer haben zwar darauf hingewiesen, dass die Steuerbelastung unterschiedlichen Entwicklungen in den Wertverhältnissen auch durch Hebesatzanpassungen Rechnung tragen kann. Dies gilt allerdings nicht für Wertanpassungen innerhalb von Gemeinden. Daher ist davon auszugehen, dass die Flächensteuer wegen des fehlenden Wertbezugs nur eine geringe Akzeptanz findet.

Um die Nutzung des Grundstücks über den bloßen Bodenwert hinaus zu berücksichtigen, könnte man dem Kompromissvorschlag des Wissenschaftlichen Beirats von 2019 folgen. Er sieht vor, dass die Bemessungsgrundlage des Bodenwerts (ermittelt aus Fläche und Bodenrichtwert) um eine additive Komponente ergänzt wird. Diese beinhaltet die Wohn- und Nutzfläche bewertet mit standardisierten Baukosten (Wissenschaftlicher Beirat beim Bundesministerium der Finanzen, 2019) ${ }^{6}$. Dieser Vorschlag geht davon aus, dass die tatsächlichen Baukosten zweitrangig sind und die Ermittlung wesentlich komplizieren würden. Gleichwohl würde die Besteuerung im Vergleich zur reinen Bodenwertsteuer deutlich komplexer. Vor allem müssten die Angaben aller Voraussicht nach vom Steuerzahler erhoben und entsprechend überprüft werden.

\section{Begrenzung der Belastung}

Ohne Einbeziehung des Gebäudeteils kommt allerdings aufgrund der einseitigen Belastung der Grundstückseigentümer der Frage eine besondere Bedeutung zu, wie eine unangemessene Belastung durch die Ausgestaltung der Grundsteuer vermieden werden kann. Zwar kann die Grundsteuer im Rahmen der Betriebskosten gegenwärtig auf die Mieter umgelegt werden. Allerdings gibt es Bestrebungen,

6 Um ein angemessenes Verhältnis der Komponenten sicherzustellen, schlägt der Beirat folgende Formel für die Bemessungsgrundlage vor: Fläche $\times$ Bodenrichtwert $/ \mathrm{m}^{2}+$ Wohn- und Nutzfläche $\times$ Baukostenwert $/$ $\mathrm{m}^{2}$. Der Baukostenwert könnte auf die durchschnittlichen Kosten zum Zeitpunkt der Genehmigung abstellen, die vom Statistischen Bundesamt in der Reihe Bauen und Wohnen bereitgestellt werden. Er wäre mit einem Preisindex fortzuschreiben, damit bei steigenden Bodenrichtwerten die Wohn- und Nutzfläche nicht an Bedeutung verliert. 
die Umlagefähigkeit der Grundsteuer abzuschaffen. Einen entsprechenden Gesetzentwurf hat das Land Berlin bereits in den Bundesrat eingebracht (Bundesrat, 2019a).

Möglicherweise schützt das Verfassungsrecht den Grundstückseigentümer vor einer „erdrosselnden Wirkung“ der Grundsteuer. Allerdings ist unklar, wann diese einsetzt. Das Bundesverwaltungsgericht hat schon 1969, als die Einheitswerte noch deutlich näher an den Verkehrswerten lagen, die Festlegung eines Hebesatzes von $1000 \%$ für rechtens erachtet (Wissenschaftliche Dienste des Deutschen Bundestags, 2016). Die Länder können aber durch Landesgesetz Höchstsätze für den Hebesatz der Grundsteuer bestimmen. Zumindest sieht dies § 26 des Grundsteuergesetzes ausdrücklich vor. Eine entsprechende Festlegung kann einen wichtigen Beitrag leisten, berechtigte Sorgen über unangemessene Belastungen zu zerstreuen. ${ }^{7}$ Im Einzelfall kann es aufgrund der Wertansätze dennoch zu einer extremen Belastung kommen. Denkbar wäre daher auch, die Steigerung der Steuerbelastung aufgrund von Neubewertungen zu begrenzen. Eine solche Vorschrift sollte allerdings nicht zu eng gefasst werden, da sie die Ergiebigkeit der Grundsteuer in Wachstumsregionen beeinträchtigt. Ohnehin würde allerdings die Einführung der auf Bodenwerte bezogenen Grundsteuer zu erheblichen Belastungsverschiebungen im Vergleich zur derzeitigen Grundsteuer führen. Dies ist aufgrund der aktuell völlig veralteten Wertansätze unvermeidlich, und Belastungsverschiebungen sind auch bei allen anderen Reformvarianten zu erwarten. Daher empfehlen sich ohnehin längere Übergangsfristen und gegebenenfalls Anpassungen zur Regelung der Stundung. Neben den hier erörterten finanzwissenschaftlichen Argumenten ist für einen Reformvorschlag wichtig, ob er auch vor der Verfassung Bestand hat. Das ist beispielsweise für den Bundesvorschlag verneint worden (Hey, 2019). Für die Bodenwertsteuer wurde argumentiert, dass trotz der Vereinfachung gegenüber anderen Modellen verfassungsrechtliche Risiken beseitigt werden (Hey, 2019).

\section{Fazit}

Eine Grundsteuer, die sich allein auf den Bodenwert bezieht, stellt eine ernstzunehmende Alternative zum Bundesvorschlag dar. Die Berücksichtigung des Grund- stückswertes bezieht indirekt auch Standortvorteile aus öffentlichen Leistungen ein. Dies ist eine wichtige Funktion der Grundsteuer. Mit den Bodenrichtwerten gibt es eine praktikable Möglichkeit, mit vergleichsweise geringem Aufwand zu belastbaren Wertansätzen zu kommen. Aus finanzwissenschaftlicher Sicht ist an einer reinen Bodenwertsteuer jedoch zu bemängeln, dass die Nutzung der Grundstücke unberücksichtigt bleibt. Zwar wäre eine fallgerechte Bewertung der Gebäude mit erheblichem Mehraufwand verbunden, der Vorschlag des Wissenschaftlichen Beirats für eine pauschalierte Bewertung anhand der Gebäudegröße ermöglicht aber auch hier eine vergleichsweise einfache Lösung. Verzichtet man auf den Gebäudeteil und basiert die Grundsteuer ausschließlich auf dem Bodenwert, sinkt der Erhebungsaufwand deutlich. Wählt der Landesgesetzgeber diesen Weg, sollten indes Vorkehrungen getroffen werden, die eine unangemessene Belastung ausschließen, beispielsweise durch Festsetzung eines Höchstsatzes für die Grundsteuer.

\section{Literatur}

Bundesrat (2019a), Bundesrats-Drucksache, 12. September, 434/19.

Bundesrat (2019b), Bundesrat beschließt Reform der Grundsteuer, Stuttgart, 8. November, https:Ilwww.baden-wuerttemberg.de/de/service/ presse/pressemitteilung/pid/bundesrat-beschliesst-reform-dergrundsteuer-2/ (31. März 2019).

Fuest, C., L. Immel, V. Meier und F. Neumeier (2018), Die Grundsteuer in Deutschland: Finanzwissenschaftliche Analyse und Reformoptionen, ifo-Institut, München, 26.

Hey, J. (2019), Grundsteuerreform 2019, Zeitschrift für Gesetzgebung, (4), 297-323.

Kronberger Kreis (2003), Gute Gemeindesteuern, Stiftung Marktwirtschaft, Berlin.

Löhr, D. (2019), Reform der Grundsteuer - Chance für eine nachhaltige Siedlungsentwicklung?, Zeitschrift für Umweltpolitik und Umweltrecht, 42.

Scheffler, W. und M. Roith (2018), Leitlinien für eine Reform der Grundsteuer, ifst-Schrift, 526, Berlin, $57 \mathrm{f}$

Van Suntum, U. und D. Schultewolter (2014), § 10 Die Besteuerung des Wohneigentums, Wohneigentum, Springer, 194.

Walczak, J. (2018), Property tax limitation regimes: A Primer, Tax Foundation, April, 23.

Wissenschaftliche Dienste des Deutschen Bundestags (2016), WD 4 $3000-023 / 16,10 \mathrm{f}$.

Wissenschaftlicher Beirat beim Bundesministerium der Finanzen (2011), Reform der Grundsteuer, Stellungnahme, Berlin.

Wissenschaftlicher Beirat beim Bundesministerium der Finanzen (2019), Die Reform der Grundsteuer - Ein Kompromissvorschlag. Stellungnahme, Berlin.

Zimmermann, H. und T. Döring (2019), Kommunalfinanzen, 4. Aufl., Berlin, 163-173.

7 Im Entwurf des Landesgrundsteuergesetzes für Baden-Württemberg ist eine Höchstgrenze allerdings nicht vorgesehen.

Title: Property-Tax Reform: The Land-Value Tax as a Practical Alternative?

Abstract: Germany is currently reforming its municipal property tax. To simplify the assessment, some German states are considering a land-value tax as a practical alternative. The authors discuss the pros and cons and find some merit in the land-value tax. However, they suggest that states implement precursions to prevent excessive taxation, such as setting a maximum tax rate.

JEL Classification: H71, R51 